\title{
Perceptions of weight and associated factors of adolescents in Jiangsu Province, China
}

\author{
Zumin Shi ${ }^{1,2, *}$, Nanna Lien ${ }^{3}$, Bernadette Nirmal Kumar ${ }^{2}$ and Gerd Holmboe-Ottesen ${ }^{2}$ \\ 'Department of Nutrition and Food Hygiene, Jiangsu Provincial Center for Disease Control and Prevention, 172 \\ Jiangsu Road, Nanjing 210009, People's Republic of China: ${ }^{2}$ Section of Preventive Medicine and Epidemiology, \\ Institute of General Practice and Community Medicine, University of Oslo, Oslo, Norway: ${ }^{3}$ Department of Nutrition, \\ University of Oslo, Oslo, Norway
}

Submitted 30 August 2005: Accepted 10 May 2006

\begin{abstract}
Objective: To describe perceptions of weight of adolescents and associated factors. Design: Cross-sectional survey in 2002.

Setting: Eight public middle schools in two distinct socio-economic areas of Jiangsu Province, China.

Subjects: Eight hundred and twenty-four adolescents (aged 12-14 years) and 628 of their parents.

Methods: Self-administered questionnaires for students and their parents were used to collect the data. Height and weight of the students were measured.

Results: One-third of the girls perceived themselves as overweight, while only 8.9\% were actually overweight or obese according to the World Health Organization definition. On the other hand, $15.0 \%$ of the girls considered themselves underweight, while $5.6 \%$ were underweight according to the definition. Among the boys, one-fifth of those defined as overweight perceived their weight as normal. Furthermore, 23.9\% perceived themselves as underweight when in fact only $4.9 \%$ were classified as underweight according to the definition. About one-quarter of the students dieted in the past year. Adolescents who perceived themselves to be overweight dieted and skipped breakfast more often. They also had lower intake frequencies of animal foods, Western foods and milk/yoghurt. No disparity was observed in the intake frequency of fruits and vegetables between the groups of different weight perception. Parents' weight norms seemed to favour overweight in both genders, but especially among boys.

Conclusions: Both overweight and underweight concerns were common among girls, while a higher body weight was favoured by normal-weight boys and their parents. Distorted weight perceptions in adolescents, as well as dieting, are problems that need appropriate intervention.
\end{abstract}

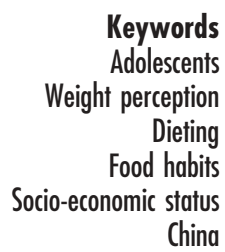

Distorted perception of weight is a worldwide problem of adolescents, especially among girls ${ }^{1-3}$. Adolescent girls are often dissatisfied with their body image, which puts them at nutritional risk because they frequently engage in dieting even when they have normal weight ${ }^{3-5}$. Studies show that peers, parents and health education professionals are influential in the development of children's body image, weight control practices, and weight-related attitudes and behaviours ${ }^{1,3,6}$. It is also clear that adolescents demonstrate significant gender and racial differences in weight perceptions and dietary behaviours ${ }^{7}$. An association was found between increased frequency of exercise, toning and stretching and trying to lose weight among females, while males trying to gain weight reported increased participation in exercise, stretching and toning and team sports ${ }^{1}$. In developed countries, adolescents' perception of themselves as overweight or normal-weight often correlate poorly with medical definitions of overweight $^{2}$. Dieting behaviour seems to be associated with whether adolescents view themselves as overweight. Normal-weight girls are more prone to consider themselves as overweight than normal-weight boys ${ }^{2,8}$. However, other family and social factors, such as parental weight, education and socio-economic status (SES), may also influence adolescent weight perceptions and dieting?

China is undergoing rapid economic growth leading to a nutrition transition. Overweight and obesity among adolescents are becoming a public health concern ${ }^{10,11}$. In parallel with this phenomenon, weight consciousness may be increasing among adolescents. The formation of weight perception is complex. Biological, psychological and sociocultural factors are found to be correlated with weight perception ${ }^{12-14}$. At present, little is known about this topic in China. However, findings from the China 
Health and Nutrition Survey revealed that some urban girls have lower energy intake than recommended, suggesting the emergence of dieting among urban girls ${ }^{15}$. There is thus a need to study lifestyle factors and their association with weight perceptions and nutritional status in China. The objectives of the present paper are to describe perceptions of weight in adolescents and the association with food habits and other lifestyle factors, as well as some sociodemographic and psychosocial factors. The research questions include: To what extent are students concerned about their body weight? What is the relationship between weight perception and actual weight? To what degree is adolescents' weight perception associated with food frequency intake, physical activity and dieting? What are parents' perceptions of their children's weight? Are there sociodemographic differences in the adolescents' perceptions and the resulting behaviour? The results will be used as a basis for health behaviour intervention.

\section{Subjects and methods}

\section{Sample, protocol and etbical approval}

A cross-sectional study was conducted in urban and rural middle schools in Jiangsu Province, China. The province is divided into 13 prefectures, which in total have 2757 junior schools with 3.25 million students ${ }^{16}$. The study locations were Zhenjiang, a rich prefecture in the southern region, and Xuzhou, a poor prefecture in the northern region. The gross domestic product of Zhenjiang is 18852 Yuan/ capita/year (\$US 2356) and in Xuzhou 7960 Yuan/capita/ year (\$US 995). In each prefecture, one school was randomly selected from each of four different areas: the capital city of the prefecture, a suburb of the capital city, a county capital and a town. In every school one junior level 1 and one junior level 2 class were selected. Thus, students from 16 classes in total were invited to join the survey. The age range of the study population was $12-14$ years, with a few exceptions. Students in special schools for the disabled or private schools were excluded. Written consent was obtained from the presidents of the schools, the teachers, the parents and the students. They were informed that answering the anonymous questionnaire was entirely voluntary and that the information would be treated confidentially.

A self-administered questionnaire was used to collect information on sociodemographic indicators, weight perception and lifestyle. The students filled in the questionnaire in the classroom within a $40 \mathrm{~min}$ period in the presence of health workers whom they could ask if they had problems. Instructions on filling in the questionnaire were given by the researcher. Another small limited questionnaire was administered to the parents to obtain among other things information about parents' weight perception and weight norms of their children. The parents' questionnaire was taken home by the students and returned to the school the following day.
The study was a collaboration between the Institute of General Practice and Community Medicine at the University of Oslo and Jiangsu Provincial Center for Disease Control and Prevention. The protocol was approved by both institutions. In addition, ethical clearance was obtained from the Regional Ethical Committee of Norway. Health workers from local centres for disease control and prevention together with the researcher carried out all data collection from September to November 2002. The questionnaire was piloted and adjusted prior to the survey.

\section{Measurements}

\section{Height and weight}

Students' height without shoes was measured to the nearest $0.1 \mathrm{~cm}$ using a Seca stadiometer, and weight in light clothes was measured to the nearest $0.1 \mathrm{~kg}$ on a Seca portable electronic scale. Each of these measurements was carried out by at least two trained health workers: one took the measurements, while a second recorded the readings. Weight status was defined according to age- and gender-specific body mass index (BMI, $\mathrm{kg} \mathrm{m}^{-2}$ ) percentiles of the US National Center for Health Statistics/World Health Organization (WHO) reference: underweight as BMI less than the 5th percentile; normal weight as BMI between the 5 th and 85 th percentile; overweight between the 85th and 95th percentile; and obesity was defined as BMI greater than the 95th percentile ${ }^{17}$.

\section{Weight perceptions and related behaviours}

Weight perceptions were assessed by the question 'What do you think about your own body weight? for students, and for the parents by the question 'What do you think about your child's body weight?' The response categories were: (1) very underweight; (2) slightly underweight; (3) about the right weight; (4) slightly overweight; (5) very overweight. In the data analysis, these responses were recoded into three categories: underweight, normal and overweight.

Weight desires among the students were assessed by the question 'Which of the following are you trying to do?', and among the parents by the question "Which of the following do you want your child to do?' The answer categories were: (1) lose weight; (2) gain weight; (3) maintain the same weight; (4) don't bother about weight.

Dieting was assessed by the question 'How often have you gone on a diet during the last year?' The response categories were: (1) never; (2) 1-4 times; (3) 5-10 times; (4) more than 10 times; (5) I am always dieting.

Weight management was measured by 'During the past 7 days, which of the following actions did you take to lose weight or to keep from gaining weight? (multiple choice)'. The response categories were: (1) dieting; (2) vomiting; (3) diet pills; (4) herbs/teas; (5) increase physical activity; (6) other method, specify; (7) not trying to lose weight. 
Vigorous physical activity was assessed by the question 'How many times per week do you work, play or exercise hard enough to make you sweat and breathe heavily?' The response categories were: (1) seldom/never; (2) 1-2 times; (3) 3 times or more.

\section{Food and meals frequency questionnaire}

We developed a food-frequency questionnaire (FFQ) including 26 food items. Portion sizes were not included. Of the questions, 16 were taken from a validated questionnaire developed by Zhao et al. ${ }^{18}$ for Chinese adults. In addition, 10 questions on snacks, fast foods and soft drinks were added. These questions were selected on the basis of knowledge from earlier research on adolescents in the province. The diet assessment question was 'How often do you eat/drink the following foods?' The response categories were (recoded frequencies in times per week shown in square brackets): (1) more than once a day [10]; (2) once a day [7]; (3) 4-6 times per week [5]; (4) 2-3 times per week [2.5]; (5) once a week [1]; (6) $1-3$ times per month [0.5]; (7) seldom/never [0]. The meal pattern assessment question was 'During an ordinary week, how often do you usually eat the following meals?' The response categories for the questions were: (1) every day; (2) 4-6 times per week; (3) 1-3 times per week; (4) seldom/never.

Five food-frequency scores were constructed as follows on the basis of selected food items taken from the FFQ.

- Fruit and vegetable score (FVS): includes fruit, vegetables (potato not included) and fruit juice.

- Animal food score (AFS): includes meat from pork, beef, lamb and chicken, as well as liver, fish, shrimps and eggs.

- Western food score (WFS): includes milk, yoghurt, soft drinks and hamburgers.

- Sweet food score (SFS): includes candy, chocolate, ice cream and soft drinks.

- Milk score (MS): includes milk and yoghurt.

The five scores (FVS, AFS, WFS, SFS and MS) were constructed by the sum of the recoded responses (in times per week) for the food items included in each score, divided by the number of items in the score. The one week test-retest reliability of the food scores among 112 participants (comprising a different sample in the same study area) had a Spearman correlation coefficient $(r)$ ranging from 0.60 to 0.82 .

\section{Sociodemographic factors}

Housebold SES score based on housebold possessions. Household SES score was the sum of ownership of a video/compact disc player, television set, personal computer, refrigerator, mobile phone, shower, washing machine, air conditioner, motorcycle, microwave oven and telephone; each item given a value of 1 . Three categories were constructed from household SES score: 'low', $\leq 4$; 'medium', 5-8; 'high', 9-11.

Educational level of parents. Educational level of the parents was reported by the students and categorised into three levels: 'low', illiteracy, primary, junior middle school; 'medium', senior middle school; 'high', university or higher.

Urban/rural. We defined those who lived in capital cities and in county capitals as 'urban', and those who lived in the capital city suburbs, towns and rural villages as 'rural'.

\section{Data analysis}

All statistical analyses were performed using SPSS 11.0 (SPSS Inc.). Statistical significance was set at $P<0.05$. Chisquare tests were used to compare frequencies. General linear model (GLM) analysis was used to compare the means of food scores among different weight perception groups. The food scores were adjusted for sociodemographic factors (household SES, urban/rural, south/north, education of father $)^{19}$ in addition to dieting. The data met the criteria for performing GLM analysis.

\section{Results}

\section{Sample characteristics}

Of the 855 students in the invited sample, 846 (99\%) participated in the study. In the analyses presented in this paper, only students aged $12-14$ years are included. Those few students who for some reasons were younger or older than this defined age group were excluded. The final sample thus consists of 824 students aged 12-14 years (amounting to 99\% of those participating in this age range), with somewhat more boys than girls (Table 1 ). The boys had higher mean height, weight and BMI than the girls. The prevalence of overweight and obesity among boys was near 18\%. In total, 628 parents completed the questionnaire giving a response rate of $76 \%$. Of the 196 non-responding parents, no difference was found in household SES and education, but $62 \%$ were from the south and $73 \%$ had male children $(P<0.001)$. Of the 628 parents' questionnaires, 98, 159 and 372 were filled in by fathers, mothers and both parents, respectively.

\section{Weight perception and weight desire}

One-third of the girls considered themselves as overweight, and 15.0\% considered themselves as underweight. The proportion of boys who perceived themselves as overweight and underweight was 22.7 and $23.9 \%$, respectively (Table 1 ). The actual prevalences of underweight were $4.9 \%$ for boys and $5.6 \%$ for girls. About the same percentage of boys and girls perceived themselves as normal-weight (53.4 vs 51.1\%). Figure 1 shows the relationship between BMI and weight perception by gender. All the girls who were overweight or obese according to the WHO definition perceived themselves as 
Table 1 Characteristics of the study population $(n=824)$

\begin{tabular}{|c|c|c|}
\hline & Boys $(n=431)$ & Girls $(n=393)$ \\
\hline Age (years), mean (SD) & $13.6(0.7)$ & $13.4(0.7)$ \\
\hline Height $(\mathrm{cm})$, mean (SD) & $159.9(8.6)$ & $156.5(5.6)$ \\
\hline Weight (kg), mean (SD) & $50.3(11.0)$ & $47.2(8.4)$ \\
\hline BMI $\left(\mathrm{kg} \mathrm{m}^{-2}\right)$, mean (SD) & $19.6(3.5)$ & $19.2(3.0)$ \\
\hline BMI percentile, mean (SD) & $48.8(29.7)$ & $47.1(27.0)$ \\
\hline \multicolumn{3}{|l|}{ Household SES* (\%) } \\
\hline Low $(\leq 4)$ & 16.7 & 13.6 \\
\hline Medium (5-8) & 53.0 & 50.4 \\
\hline High $(9-11)$ & 30.2 & 36.1 \\
\hline \multicolumn{3}{|l|}{ Region (\%) } \\
\hline South (Źhenjiang) & 49.2 & 51.7 \\
\hline North (Xuzhou) & 50.8 & 48.3 \\
\hline Urban (\%) & 52.0 & 56.7 \\
\hline \multicolumn{3}{|l|}{ Weight status† (\%) } \\
\hline Underweight & 4.9 & 5.6 \\
\hline Overweight & 10.9 & 6.4 \\
\hline Obese & 7.0 & 2.5 \\
\hline \multicolumn{3}{|l|}{ Weight perception (\%) } \\
\hline Underweight & 23.9 & 15.0 \\
\hline Normal & 53.4 & 51.1 \\
\hline Overweight & 22.7 & 33.8 \\
\hline
\end{tabular}

SD - standard deviation; BMI - body mass index; SES - socio-economic status.

*Household SES score based on the possession of 11 household amenities; each item given a value of 1 .

†Defined on the basis of age- and sex-specific BMI percentiles of the National Center for Health Statistics/World Health Organization reference: less than 5th as 'underweight', higher than 85th as 'overweight', higher than 95th as 'obese'.

overweight. In addition, about $30 \%$ of the girls with normal weight also perceived themselves as overweight. One-fifth of the overweight or obese boys perceived their weight as normal. Among the adolescents with normal weight, about $12 \%$ of the girls and $25 \%$ of the boys perceived themselves as underweight.

Within the same BMI groups no differences were observed in weight perception by household SES, education of parents or urban/rural location. However, more boys in the south perceived themselves as underweight than boys in the north (31.6 vs. 16.4\%). No significant differences were observed in weight perception between girls from the north and the south (data not shown).
On average, 34\% of the students wanted to lose weight, while $18.1 \%$ wanted to gain weight. More girls wanted to lose weight than boys ( 45 vs. 24\%) (data not shown). Half of the urban girls wanted to lose weight, compared with $39 \%$ of the rural girls $(P<0.05)$ (data not shown). More than $40 \%$ of the girls wanted to lose weight even if they had a normal weight (Table 2). In contrast to the girls, $29.8 \%$ of boys wanted to gain weight even if they had a normal weight. Some $45.5 \%$ of the girls who were underweight wanted to maintain the same weight or did not bother about weight.

The weight perception of the students was strongly correlated with the desire to change weight (Spearman $r=0.67 ; P<0.001$ ), and perceiving oneself as overweight was positively correlated to wanting to lose weight.

\section{Parents' weight perceptions and weight norms}

About $22 \%$ of the parents regarded their child as underweight even if their child had a normal weight. Of the overweight children, 23\% were perceived as having normal weight by their parents. Unlike the children, only $55.2 \%$ of the parents wanted the overweight children to lose weight. One-quarter of the parents wanted their normalweight children to gain weight. Parents' perceptions of children's weight differed by the child's gender. Compared with girls, a higher percentage of normal-weight boys were perceived as underweight (29.5 vs. $15.6 \%$ ) by their parents and were desired to gain weight (34.6 vs. 16.7\%) (Table 3). Urban/rural, south/north, household SES and education were not associated with parents' perceptions and norms of their child's weight (data not shown).

\section{Dieting and weight control measures}

During the last year, $24.2 \%$ of the students had been on a diet, $13.6 \%$ of them had been on a diet $1-4$ times and $6.3 \%$ had been on a diet more than 10 times. The girls were more likely to go on a diet than the boys (29.8 vs. 19.1\%). Among the 280 (34\%) students who wanted to lose weight, $45.4 \%$ had been dieting during the past year; among those
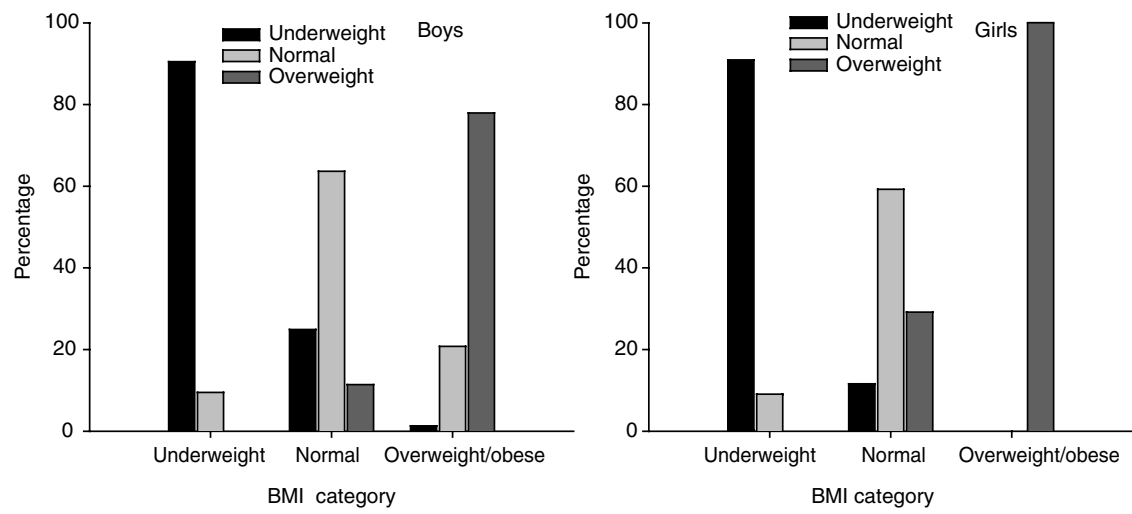

Fig. 1 Relationship between body mass index (BMI) and weight perception by gender in a sample of Chinese adolescents from Jiangsu Province (boys $n=431$, girls $n=393$ ). BMI categories were defined on the basis of age- and sex-specific BMI percentiles of the National Center for Health Statistics/World Health Organization reference: less than 5th as 'underweight', higher than 85th as 'overweight', higher than 95th as 'obese' 
Table 2 Desire to change weight by gender and BMI category* in a sample of Chinese adolescents from Jiangsu Province $(n=824)$

\begin{tabular}{lcccc}
\hline & Lose weight & Gain weight & $\begin{array}{c}\text { Maintain the same/Don't } \\
\text { bother about weight }\end{array}$ & $P$-value† \\
\hline Boys & & & & \\
$\quad$ Underweight $(n=21)$ & 4.8 & 76.2 & 19.1 & $<0.001$ \\
$\quad$ Normal $(n=333)$ & 13.0 & 29.8 & 57.2 & \\
$\quad$ Overweight/obese $(n=77)$ & 77.9 & 1.3 & 20.7 & \\
Girls & & & & \\
$\quad$ Underweight $(n=22)$ & 0 & 54.5 & 45.5 & \\
$\quad$ Normal $(n=336)$ & 43.5 & 6.3 & 50.3 & \\
$\quad$ Overweight/obese $(n=35)$ & 85.7 & 0 & 14.2 & \\
Total & 34.0 & 18.1 & 47.9 & \\
\end{tabular}

$\mathrm{BMI}$ - body mass index.

* Defined on the basis of age- and sex-specific BMI percentiles of the National Center for Health Statistics/ World Health Organization reference: less than 5th as 'underweight', higher than 85th as 'overweight', higher than 95th as 'obese'.

†Fisher's exact test.

there were more girls (50.0\%) than boys (37.5\%). Within the genders, there was no urban/rural, south/north or household SES difference in the prevalence of dieting (data not shown).

Table 3 Comparison of child's BMI category* with parent's weight perception and weight desire for their child in a sample of Chinese adolescents from Jiangsu Province (boys $n=431$, girls $n=393$ )

\begin{tabular}{|c|c|c|c|}
\hline & \multicolumn{3}{|c|}{ Child's BMI category } \\
\hline & $\begin{array}{l}\text { Underweight } \\
\qquad(n=31)\end{array}$ & $\begin{array}{l}\text { Normal } \\
(n=530)\end{array}$ & $\begin{array}{c}\text { Overweight/obese } \\
\qquad(n=87)\end{array}$ \\
\hline \multicolumn{4}{|c|}{ Parent's weight perception of their child (\%) } \\
\hline \multicolumn{4}{|c|}{ Underweight } \\
\hline Boys & 85.7 & 29.5 & 1.6 \\
\hline Girls & 94.1 & 15.6 & 0 \\
\hline Both sexes & 90.3 & 22.0 & 1.2 \\
\hline \multicolumn{4}{|l|}{ Normal } \\
\hline Boys & 14.3 & 66.2 & 27.4 \\
\hline Girls & 5.9 & 72.1 & 12.0 \\
\hline Both sexes & 9.7 & 69.4 & 23.0 \\
\hline \multicolumn{4}{|l|}{ Overweight } \\
\hline Boys & 0 & 4.3 & 71.0 \\
\hline Girls & 0 & 12.3 & 88.0 \\
\hline Both sexes & $\begin{array}{l}0 \\
0-0131+\end{array}$ & $\begin{array}{c}8.6 \\
0<0\end{array}$ & $\begin{array}{c}75.8 \\
P=0.31\end{array}$ \\
\hline \multirow{2}{*}{\multicolumn{4}{|c|}{ Parent's weight desire for their child (\%) }} \\
\hline & & & \\
\hline $\begin{array}{l}\text { Loys } \\
\text { Boys }\end{array}$ & \multicolumn{3}{|c|}{ Lose weight } \\
\hline Girls & 0 & $\begin{array}{l}1.3 \\
7.6\end{array}$ & $\begin{array}{l}51.0 \\
64.0\end{array}$ \\
\hline Both sexes & 0 & 4.7 & 55.2 \\
\hline \multicolumn{4}{|l|}{ Gain weight } \\
\hline Boys & 85.7 & 34.6 & 3.2 \\
\hline Girls & 88.2 & 16.7 & 0 \\
\hline Both sexes & 87.1 & 24.9 & 2.3 \\
\hline \multicolumn{4}{|c|}{ Maintain/do not bother } \\
\hline Boys & 14.3 & 64.1 & 45.2 \\
\hline Girls & 11.8 & 75.7 & 36.0 \\
\hline \multirow[t]{2}{*}{ Both sexes } & 12.9 & 70.4 & 42.5 \\
\hline & $P=0.835$ & $P<0.001$ & $P=0.437$ \\
\hline
\end{tabular}

BMI - body mass index.

* Defined on the basis of age- and sex-specific BMI percentiles of the National Center for Health Statistics/World Health Organization reference: less than 5th as 'underweight', higher than 85th as 'overweight', higher than 95th as 'obese'.

$\dagger P$-value for the comparison (chi-square test) between parent's weight perception/weight desire and child's gender within the same BMI category of child.
Dieting was associated with weight perception, $40.6 \%$ of the girls and $31.6 \%$ of the boys who perceived themselves as overweight reported to be dieting. About $27 \%$ of the girls who reported to have been dieting during the past year perceived their body weight to be normal (Table 4).

Among the students who wanted to lose weight, 23.6\% had been dieting and 69\% adopted exercise as a measure to control weight during the past 7 days. Less than $2 \%$ took dieting pills, herbs or tea, or practised vomiting to control weight (data not shown).

The boys who reported dieting had lower AFS (mean (standard deviation, SD): 1.9 (1.1) for dieters, 2.3 (1.4) for non-dieters, $P<0.05)$, while the girls who went on a diet had lower SFS (mean (SD): 2.0 (1.9) for dieters, 2.7 (2.3) for non-dieters, $P<0.01)$. There was no difference in the other food scores between dieting and non-dieting adolescents.

Less than half of the adolescents engaged in vigorous physical activity 3 times or more per week. No association was found between weight perception and vigorous physical activity (Table 4).

Table 4 Association between weight perception and lifestyle factors in a sample of Chinese adolescents from Jiangsu Province (boys $n=431$, girls $n=393)(\%)$

\begin{tabular}{|c|c|c|c|c|}
\hline & \multicolumn{3}{|c|}{ Weight perception } & \multirow[b]{2}{*}{$P$-value* } \\
\hline & Underweight & Normal & Overweight & \\
\hline \multicolumn{5}{|c|}{ Dieting last year } \\
\hline Boys & 12.6 & 16.6 & 31.6 & 0.001 \\
\hline Girls & 13.6 & 27.4 & 40.6 & $<0.001$ \\
\hline Both sexes & 13.0 & 21.6 & 36.8 & $<0.001$ \\
\hline \multicolumn{5}{|c|}{ Breakfast $<3$ times/week } \\
\hline Boys & 3.9 & 5.7 & 10.2 & 0.155 \\
\hline Girls & 5.1 & 3.5 & 9.0 & 0.096 \\
\hline Both sexes & 4.3 & 4.6 & 9.5 & 0.025 \\
\hline \multicolumn{5}{|c|}{ VPA $>3$ times/week } \\
\hline Boys & 46.1 & 49.1 & 52.0 & 0.701 \\
\hline Girls & 39.0 & 37.8 & 46.6 & 0.262 \\
\hline Both sexes & 43.5 & 43.9 & 48.9 & 0.407 \\
\hline
\end{tabular}

VPA - vigorous physical activity.

${ }^{*}$ Chi-square test. 


\section{Association between weight perception and meal pattern and food frequency intake}

Table 4 shows that weight perception was associated with infrequent breakfast consumption ( $\leq 3$ times/week). In total, 9.5\% of the adolescents who perceived themselves as overweight skipped breakfast, compared with less than $5 \%$ of adolescents who perceived themselves as normalweight or underweight.

No association was found between BMI and food scores (data not shown). Table 5 shows the comparison of food scores by weight perception adjusted for sociodemographic factors and dieting. Both boys and girls who perceived themselves as overweight reported a lower frequency of intake of animal foods, Western foods and dairy foods. The SFS was associated with weight perception only among boys. Weight perception was not associated with fruits and vegetables intake in either gender.

\section{Discussion}

Results from a cross-sectional study of weight perception and the association with food habits and other lifestyle factors in a subgroup of adolescents aged 12-14 years from Jiangsu Province are reported. A quite high percentage of the girls perceived themselves as overweight even if they had a normal weight. In contrast to the girls, more boys wanted to gain weight. Parents' weight norms seemed to favour overweight, especially for boys. About one-quarter of the students had been on a diet during the past year. Sociodemographic factors were not associated with weight perception within the same BMI category. Perception of overweight was positively associated with breakfast skipping and dieting, and negatively associated with frequency intake of animal foods, Western foods and milk/yoghurt. The findings reveal that distorted weight perception is common, especially among girls.

It is clear from the results of the current study that a conscious weight perception and weight concerns are present in adolescents aged 12-14 years in China. Both overweight and underweight concerns have public health importance. About 30\% thought of themselves as overweight. Similar percentages of boys and girls were satisfied with their body weight. A quite high percentage (about $30 \%$ ) of the girls perceived themselves as overweight even if they had a normal weight. Of particular concern is that one-fifth of the overweight boys perceived their weight as normal while one-quarter of the normal-weight boys perceived themselves as underweight. About 12\% of the normal-weight girls perceived themselves as underweight. Similar results on weight perception have been found in many Western countries ${ }^{1,2,7,9}$.

In Western countries, higher SES is found to be associated with greater weight satisfaction?. We did not find such association in our study. Within the same BMI groups no differences were observed between weight perception and household SES or urban/rural location.

Our results showed that one-third of the students wanted to lose weight. Of particular concern is that more than $40 \%$ of the normal-weight girls wanted to lose weight. In contrast to the girls, the boys wanted to gain weight even if they had a normal weight. This gender difference has been reported in many developed countries ${ }^{3,6,7}$. The girls were nearly twice as likely as the boys to want to weigh less. Girls in urban areas were more weight-conscious than rural girls. Nearly half of the urban girls wanted to lose weight. This number is comparable to that of adolescent girls in Western countries $^{1,3}$. The fact that no difference was found in prevalence of dieting between urban and rural girls is surprising, since this has been found in another study on

Table 5 Comparison* of food scores by weight perception in a sample of Chinese adolescents from Jiangsu Province $(n=824)$

\begin{tabular}{|c|c|c|c|c|c|c|c|c|c|c|}
\hline \multirow[b]{2}{*}{ Weight perception } & \multicolumn{2}{|c|}{ AFS† } & \multicolumn{2}{|c|}{ WFS $\ddagger$} & \multicolumn{2}{|c|}{ SFS§ } & \multicolumn{2}{|c|}{ MSף } & \multicolumn{2}{|c|}{ FVS\| } \\
\hline & Mean & SE & Mean & SE & Mean & SE & Mean & SE & Mean & SE \\
\hline \multicolumn{11}{|l|}{ Boys } \\
\hline Underweight $(n=103)$ & 2.8 & 0.2 & 2.7 & 0.2 & 1.7 & 0.2 & 4.1 & 0.3 & 5.0 & 0.2 \\
\hline Normal $(n=230)$ & 2.9 & 0.1 & 3.1 & 0.1 & 2.3 & 0.1 & 4.2 & 0.2 & 5.1 & 0.1 \\
\hline Overweight $(n=98)$ & 2.4 & 0.1 & 2.4 & 0.2 & 1.6 & 0.2 & 3.2 & 0.3 & 4.8 & 0.2 \\
\hline$P$-value ${ }^{\star *}$ & \multicolumn{2}{|c|}{0.014} & \multicolumn{2}{|c|}{0.011} & \multicolumn{2}{|c|}{0.009} & \multicolumn{2}{|c|}{0.016} & \multirow{2}{*}{\multicolumn{2}{|c|}{0.392}} \\
\hline \multicolumn{9}{|l|}{ Girls } & & \\
\hline Underweight $(n=59)$ & 2.1 & 0.1 & 2.8 & 0.2 & 2.1 & 0.3 & 4.3 & 0.3 & 5.4 & 0.3 \\
\hline Normal $(n=201)$ & 2.3 & 0.1 & 2.9 & 0.1 & 2.4 & 0.1 & 4.1 & 0.2 & 5.6 & 0.1 \\
\hline Overweight $(n=133)$ & 1.9 & 0.1 & 2.4 & 0.1 & 2.0 & 0.2 & 3.4 & 0.2 & 5.7 & 0.2 \\
\hline$P$-value ${ }^{\star \star}$ & \multicolumn{2}{|c|}{0.072} & \multicolumn{2}{|c|}{0.041} & \multicolumn{2}{|c|}{0.263} & \multicolumn{2}{|c|}{0.028} & \multicolumn{2}{|c|}{0.504} \\
\hline
\end{tabular}

SE - standard error.

* General linear model analysis was used to compare the means. The means were adjusted for household socio-economic status, urban/rural, south/north, education of father, age and dieting

† Animal food score: includes meat, beef, lamb, chicken, liver, fish, shrimps and eggs.

$\ddagger$ Western food score: includes milk, yoghurt, soft drinks and hamburgers.

$\S$ Sweet food score: includes candy, chocolate, ice cream and soft drinks.

I Milk score: includes milk and yoghurt.

|| Fruit and vegetable score: includes fruits, vegetables and juice.

${ }^{* *} P$-value for the comparison of food score by weight perception adjusted for other factors. 
urban/rural differences ${ }^{15}$. This necessitates further research on factors governing the relationship between weight perception and dieting in China.

Other studies from the USA have also shown that overweight boys are relatively unconcerned about their physical appearance and not particularly interested in pursuing weight loss ${ }^{7,20}$. In the present study, $20.7 \%$ of the boys having BMI higher than the 85th percentile did not want to lose weight. Public health workers should be aware of this fact when designing intervention programmes targeting overweight/obesity.

Parents' weight norms were different from the children's; one-quarter of the parents wanted their normal-weight children to gain weight, $42.5 \%$ of the parents wanted their overweight children to maintain weight or they did not bother about their weight. According to tradition, parents think the heavier child is healthier. Being overweight is favoured, especially for boys, as illustrated by the Chinese term panxiaozi (obese boy) used to appraise a boy's chubby appearance. Interestingly, no association was found between parents' weight norms and children's food scores. These results should be of particular concern for health professionals. The fact that no association was found between parents' education and their weight norms might suggest a general need to educate the parents about 'normal' weight.

The most common strategies for losing weight were dieting and increasing physical activity. Of the adolescents who viewed themselves as overweight, 39.8\% reported to having dieted during the past year. Dieting among adolescents is thus quite common also among Chinese adolescents. Although dieting represents a potential solution to obesity, it may have negative health effects ${ }^{21,22}$. It is reported that adolescents may have their own understanding and definition of dieting ${ }^{23}$. In our data, dieting boys reported lower animal food score while dieting girls reported lower sweet food scores. For this reason we adjusted for dieting while comparing the means of food scores among different weight perception groups. Further research is needed in this area as in our study we only have self-reported information on dieting instead of the amount of food intake.

About $70 \%$ of those who wanted to lose weight adopted physical exercise as a measure to control weight. This is an optimistic aspect as it showed that the students were aware of the relationship between physical activity and weight control. However, the weak association between weight perception and vigorous physical activity (as indicated in Table 4) may show the need to motivate students to increase physical activity by offering activities that fit with their actual weight. Less than $2 \%$ took dieting pills, herbs or teas, or practised vomiting. This may imply that the level of awareness of such alternative methods is low in the area at present, but this may change with the process of modernisation.

In Western countries adolescents who are trying to control their weight concentrate on eliminating high-fat foods from their diets ${ }^{24,25}$. Similar results were found in the present study. Both boys and girls having overweight perception had lower frequency intake of animal foods and Western foods. This may suggest that many adolescents seem to be aware of the high content of fat in these foods.

Many studies show that adolescents adopt increasing consumption of fruit and vegetables as one measure of weight control ${ }^{24,26}$. In our study, however, we did not find an association between weight perception and consumption of fruits/vegetables. One explanation could be that the present fruit and vegetable consumption is high among these adolescents and the variance is small.

When trying to lose weight, adolescents in Western countries often reduce the consumption of sweet foods ${ }^{1,24}$. Our results that showed boys having an overweight weight perception had lower frequency intake of sweet foods than the others. There was no association between weight perception and sweet food score among girls. Further research is needed.

The prevalence of overweight/obesity in the sample was not high, but the prevalence of obesity was higher among adolescents from urban, high-SES and highly educated fathers ${ }^{11}$. This may show the potential for increased obesity in the future if China continues to develop economically and urbanise further. The present findings showed that adolescent girls' weight perception and its related food habits were in favour of preventing obesity. Yet, the prevalence of overweight/obesity $\left(\mathrm{BMI}>24 \mathrm{~kg} \mathrm{~m}^{-2}\right.$ ) among adult women above the age of 45 years is high in the province, especially in the urban area where it is above $67 \%^{27}$. To what extent these girls become obese as adults, and how, are issues of interest. There is a need for further research into the transition of weight perception and its health implication from adolescence to mature age.

The study has some limitations. The FFQ was tested for reliability (a one week test-retest) but not for validity, although many of the questions used had been validated for adults as described elsewhere ${ }^{19}$. Questions on weight perception and physical activity have not been validated in China although the same questions have commonly been used in Western countries ${ }^{7,28}$. As we focused on sociodemographic differences, the selected sample may not be representative of the general population. The strength of the study is that height and weight of adolescents were measured.

\section{Conclusion}

Health workers need to know that distorted perceptions of weight exist among students and their parents in the province. Overweight and underweight concerns among the girls, and the favouring of overweight among the boys and the parents, should be dealt with at the same time. Overweight perception is associated with a lower intake 
frequency of fatty foods, but is not associated with intake of fruits and vegetables. Dieting and increased physical activity are the main strategies for losing weight and appropriate interventions for the prevention of obesity and overweight should be designed that build on the positive elements of these strategies, which at the same time should be sensitive to the danger of creating eating disorders among the adolescents. Interventions aimed at creating more realistic perceptions of 'normal' weight seem to be needed. Interventions should be integrated within the current health education curriculum, and also include a component aimed at parents.

\section{Acknowledgements}

Sources of funding: This study was funded by the Norwegian Directorate of Health and Social affairs under the auspices of the Norway-China Health Agreement.

Conflict of interest declaration: None declared.

Authorship responsibilities: Z.S., N.L., B.N.K. and G.H.-O. contributed to the conception and design of the study. Z.S. collected and analysed the data, and all authors contributed to the interpreration of the data. Z.S. drafted the paper and N.L., B.N.K. and G.H.-O. commented upon and revised drafts. All authors gave their approval to the final version submitted for publications.

Acknowledgements: We would like to thank the participating regional centres for disease control and prevention in Jiangsu Province, including Zhenjiang, Xuzhou, Danyang and Tongshan Centres, for their support to the data collection.

\section{References}

1 Middleman AB, Vazquez I, Durant RH. Eating patterns, physical activity, and attempts to change weight among adolescents. Journal of Adolescent Health 1998; 22(1): 37-42.

2 Strauss RS. Self-reported weight status and dieting in a crosssectional sample of young adolescents: National Health and Nutrition Examination Survey III. Archives of Pediatrics \& Adolescent Medicine 1999; 153(7): 741-7.

3 Kaneko K, Kiriike N, Ikenaga K, Miyawaki D, Yamagami S. Weight and shape concerns and dieting behaviours among pre-adolescents and adolescents in Japan. Psychiatry and Clinical Neurosciences 1999; 53(3): 365-71.

4 Fung MS, Yuen M. Body image and eating attitudes among adolescent Chinese girls in Hong Kong. Perceptual and Motor Skills 2003; 96(1): 57-66.

5 McGuire MT, Story M, Neumark-Sztainer D, Halcon L, Campbell-Forrester S, Blum RW. Prevalence and correlates of weight-control behaviors among Caribbean adolescent students. Journal of Adolescent Health 2002; 31(2): 208-11.

6 Neumark-Sztainer D, Story M, Hannan PJ, Perry CL, Irving LM. Weight-related concerns and behaviors among overweight and nonoverweight adolescents: implications for preventing weight-related disorders. Archives of Pediatrics $\mathcal{E}$ Adolescent Medicine 2002; 156(2): 171-8.

7 O'Dea JA, Caputi P. Association between socioeconomic status, weight, age and gender, and the body image and weight control practices of 6- to 19-year-old children and adolescents. Health Education Research 2001; 16(5): 521-32.
8 Roberts SJ, McGuiness PJ, Bilton RF, Maxwell SM. Dieting behavior among 11-15-year-old girls in Merseyside and the Northwest of England. Journal of Adolescent Health 1999; 25(1): 62-7.

9 Story M, French SA, Resnick MD, Blum RW. Ethnic/racial and socioeconomic differences in dieting behaviors and body image perceptions in adolescents. International Journal of Eating Disorders 1995; 18(2): 173-9.

$10 \mathrm{Ji}$ CY. Investigations on the changes of growth and nutritional status of Chinese youths, and the improving strategies and measures upon them. Journal of Peking University [Health Sciences] 2002; 34: 525-9.

11 Shi Z, Lien N, Kumar BN, Dalen I, Holmboe-Ottesen G. The socio-demographic correlates of nutritional status of school adolescents in Jiangsu Province, China. Journal of Adolescent Health 2005; 37(4): 313-22.

12 Ricciardelli LA, McCabe MP. Children's body image concerns and eating disturbance: a review of the literature. Clinical Psychology Review 2001; 21(3): 325-44.

13 McCabe MP, Ricciardelli LA. Sociocultural influences on body image and body changes among adolescent boys and girls. Journal of Social Psychology 2003; 143(1): 5-26.

14 Ricciardelli LA, McCabe MP, Banfield S. Sociocultural influences on body image and body change methods. Journal of Adolescent Health 2000; 26(1): 3-4.

15 Wang Y, Popkin B, Zhai F. The nutritional status and dietary pattern of Chinese adolescents, 1991 and 1993. European Journal of Clinical Nutrition 1998; 52(12): 908-16.

16 Jiangsu Bureau of Statistics. Statistical Yearbook of Jiangsu. Beijing: China Statistics Press, 2002.

17 Kuczmarski RJ, Ogden CL, Grummer-Strawn LM, Flegal KM, Guo SS, Wei R, et al. CDC growth charts: United States. Advance Data 2000; (314): 1-27.

18 Zhao W, Hasegawa K, Chen J. The use of food-frequency questionnaires for various purposes in China. Public Health Nutrition 2002; 5(6A): 829-33.

19 Shi Z, Lien N, Kumar BN, Holmboe-Ottesen G. Sociodemographic differences in food habits and preferences of school adolescents in Jiangsu Province, China. European Journal of Clinical Nutrition 2005; 59(12): 1439-48.

20 Steen SN, Wadden TA, Foster GD, Andersen RE. Are obese adolescent boys ignoring an important health risk? International Journal of Eating Disorders 1996; 20(3): 281-6.

21 Lowe MR, Timko CA. Dieting: really harmful, merely ineffective or actually helpful? British Journal of Nutrition 2004; 92(Suppl. 1): S19-22.

22 French SA, Jeffery RW. Consequences of dieting to lose weight: effects on physical and mental health. Health Psychology 1994; 13(3): 195-212.

23 Roberts SJ, Maxwell SM, Bagnall G, Bilton R. The incidence of dieting amongst adolescent girls: a question of interpretation? Journal of Human Nutrition and Dietetics 2001; 14(2): 103-9.

24 Nowak M. The weight-conscious adolescent: body image, food intake, and weight-related behavior. Journal of Adolescent Health 1998; 23(6): 389-98.

25 French SA, Perry CL, Leon GR, Fulkerson JA. Dieting behaviors and weight change history in female adolescents. Health Psychology 1995; 14(6): 548-55.

26 Fulkerson JA, French SA, Story M. Adolescents' attitudes about and consumption of low-fat foods: associations with sex and weight-control behaviors. Journal of the American Dietetic Association 2004; 104(2): 233-7.

27 Pan XQ, Yuan BJ, Shi Z, Dai Y. The distribution of body mass index among adults in Jiangsu Province (in Chinese). Jiangsu Preventive Medicine 2004; 15(3): 13-4.

28 Neumark-Sztainer D, Story M, Toporoff E, Himes JH, Resnick MD, Blum RW. Covariations of eating behaviors with other health-related behaviors among adolescents. Journal of Adolescent Health 1997; 20(6): 450-8. 